\title{
DOMÓTICA MEDIANTE LA TRANSMISIÓN DE DATOS DIGITALES POR LA RED ELÉCTRICA USANDO PROTOCOLO X10
}

\author{
DOMOTICS BY DIGITAL DATA TRANSMITTING \\ ON THE ELECTRICAL NETWORK USING PROTOCOL X10
}

Freedy Sotelo Valer*

Arturo Cubas Rodríguez**

RECEPCIÓN: MAYO DE 2017

ACEPTACIÓN: JULIO DE 2017

\begin{abstract}
RESUMEN
El presente ensayo describe una aplicación de la domótica muy en boga en la actualidad, que consiste en el control de una casa inteligente mediante la tecnología PLC (Power Line Comunication), la misma que hace uso del protocolo X10, un estándar de comunicación para transmitir señales de control entre equipos de automatización del hogar a través de la red eléctrica. Se propone un modelo físico que ayuda a comprender el modo en que el sistema trabaja y se analizan las partes de emisión y recepción, necesarios para la comunicación.
\end{abstract}

Palabras clave: X10, domótica, PLC, red eléctrica.

\begin{abstract}
This essay describes an application of today's most fashionable Home Automation, which controls an intelligent home using PLC (Power Line Communication) technology that makes use of the $\mathrm{X} 10$ protocol which is a communication standard for transmitting signals of control between automation equipment of the home through the electric network. It gives a physical model that helps to understand how the system works and analyze the parts of emission and reception, necessary for communication.
\end{abstract}

Keywords: X10, Home automation, PLC, Electrical network.

\footnotetext{
* Docente. Universidad Ricardo Palma. <fresov@hotmail.com>

** Bachiller Ingeniería Mecatrónica. <19981023@uni.edu.pe>
} 


\section{Introducción}

\section{Power Line Comunication - PLC}

La tecnología PLC (Power Line Comunication) portadora de red X10 es "un estándar de comunicación para transmitir señales de control entre equipos de automatización a través de la red eléctrica. Es un protocolo estandarizado que no se necesita instalar cables adicionales" [1]. En la figura 1 se muestra diagrama del sistema.

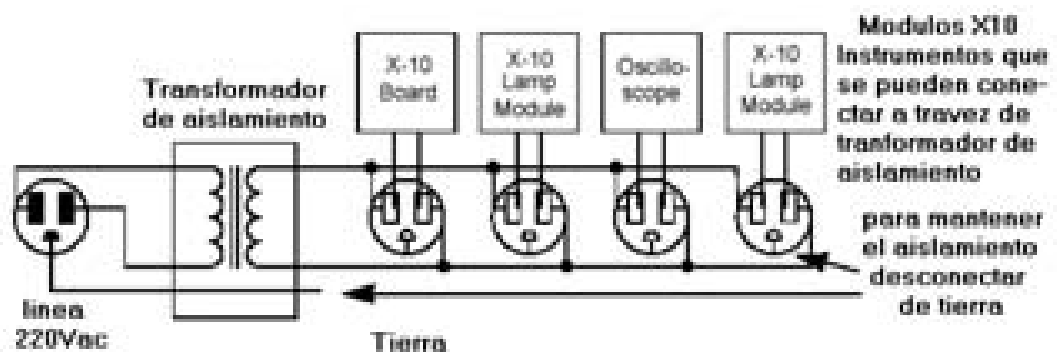

Fig. 1. Equipos X10 conectados a la red.

Fuente: Paz, H. 2003. Transmisión de información a través de la red eléctrica domiciliaria.

\section{Protocolo de comunicación $\times 10$}

El X10 es un protocolo de comunicación que permite "controlar aparatos eléctricos a través de la instalación de red eléctrica” [1]. La transmisión de una señal binaria en X10 se realiza mediante ráfagas de $120 \mathrm{KHz}$ superpuestas en los cruces por cero de la señal de la red eléctrica. En la figura 2, se muestra la forma de onda de la señal eléctrica y la comparación con las ráfagas de $120 \mathrm{KHz}$.

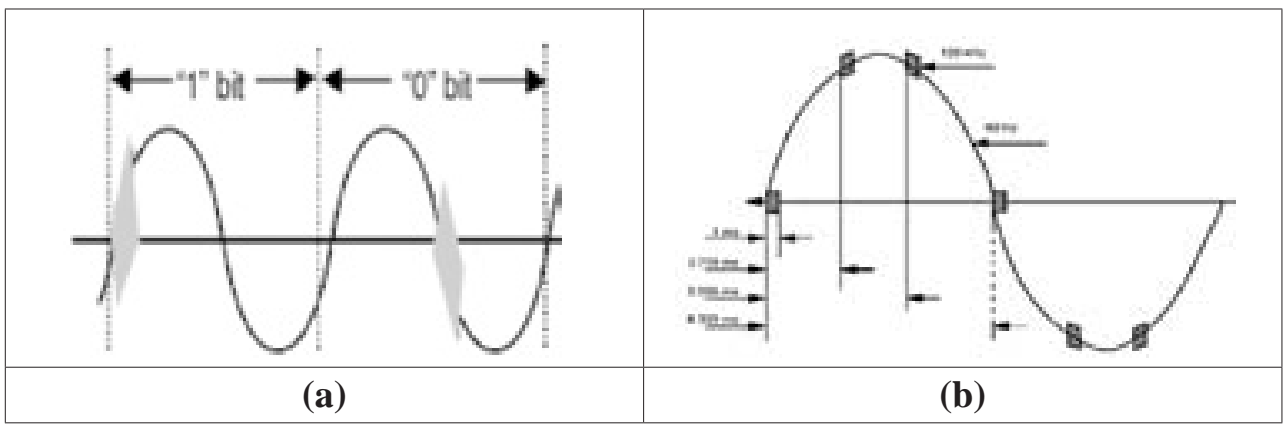

Fig. 2. (a) Señal de red eléctrica; (b) esquema de transmisión de protocolo X10.

Fuente: Paz, H. 2003. Transmisión de información a través de la red eléctrica domiciliaria.

El transceiver X10 está pendiente de los pasos por cero de la onda senoidal de $60 \mathrm{~Hz}$, típica de la alimentación eléctrica, para insertar, un instante después, una ráfaga muy corta de señal en una frecuencia fija. "Se puede insertar esta seńal en el semiciclo positivo y el negativo de la onda senoidal. La codificación de un bit 1 o de un bit 0 depende de cómo se inyecte esta señal en los dos semiciclos" [1].

Un 1 binario se representa por un pulso de $120 \mathrm{KHz}$ durante 1 milisegundo y el 0 binario se representa por la ausencia de ese pulso de $120 \mathrm{KHz}$. En un sistema trifásico, el pulso de 1 milisegundo se transmite tres veces para que coincida con el paso por el cero en las tres fases. 
Con la presencia de un pulso en un semiciclo y la ausencia del mismo en el semiciclo siguiente, se representa un ' 1 ' lógico y, a la inversa, se representa un ' 0 '. A su vez, cada comando se transmite dos veces, con lo cual toda la información transmitida tiene cuádruple redundancia. De esta manera, la comunicación es eficaz, inclusive en líneas de tensión que tengan ruido” [2].

El tiempo de bit coincide con los 16.66 ms que dura el ciclo de la señal, de forma que la velocidad binaria de 60 bps viene impuesta por la frecuencia de la red eléctrica. La transmisión completa de una orden X10 necesita once ciclos de corriente. Esta trama se divide en tres campos de información:

1. Dos ciclos representan Código de Inicio.

2. Cuatro ciclos representan Código de Casa (letras A-P),

3. Cinco ciclos representan el Código Numérico (1-16) o el Código de Función (Encender, Apagar, Aumento de Intensidad, etc.).

Por lo tanto, cada comando involucra 11 ciclos de red $(182.66 \mathrm{~ms})$. Lo anterior se muestra en la figura 3.
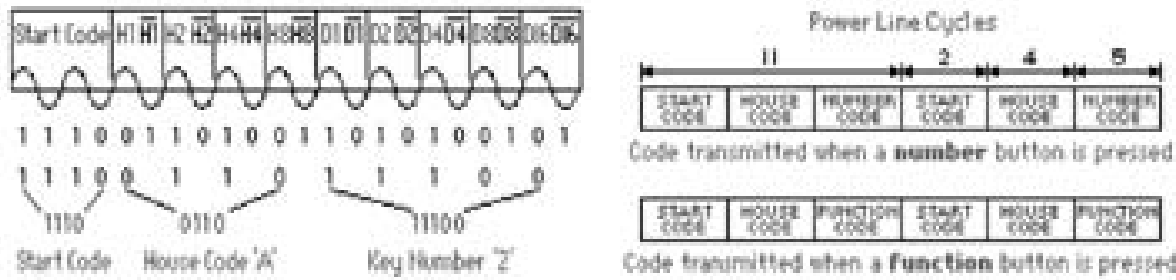

Fig. 3. Trama de orden en protocolo X10.

Fuente: http://www.qsl.net/ea3cno/pdf/articulo_x10.pdf

\section{Implementación}

\section{Consideraciones técnicas}

Para aumentar la fiabilidad del sistema, la trama (Código de Inicio, Código de Casa y Código de Función o Numérico) se transmite siempre dos veces separadas por tres ciclos completos de corriente. Hay una excepción: en funciones de regulación de intensidad, se transmiten de forma continuada (por lo menos dos veces) sin separación entre tramas.

Hay tres tipos de dispositivos X10: los que solo pueden transmitir órdenes, los que solo pueden recibirlas y los que pueden enviar o recibir estas órdenes. Como ya se dijo, primero, se transmite el código de inicio (1110), un comando con el código local (House Code) y el número de módulo (Number Code) que direcciona el módulo en cuestión. Luego, se transmite otro comando con el código de función a realizar (Function Code). Los códigos de casa (House Codes) se transmiten de acuerdo con la tabla 1 .

Los transmisores (transmitters) envían comandos X10 codificados como una señal de baja intensidad que se superpone a la señal de la red. Además, vienen dotados de dos pequeños conmutadores giratorios: uno con 16 letras (House Addresses) y el otro con 16 números (Unit Addresses) que permiten asignar una dirección de las 256 posibles. Los receptores (receivers) son capaces de demodular la señal y, si corresponde con su dirección, actuar en consecuencia. En una misma instalación, puede haber varios receptores configurados con la misma dirección: todos realizarán la función preasignada cuando un transmisor envíe una trama con esa dirección. 
TABLA 1. CÓdigos DE CASA

\begin{tabular}{|c|c|c|c|c|}
\hline House & & House & Codes & \\
\hline Addresses & H1 & H2 & H4 & H8 \\
\hline A & 0 & 1 & 1 & 0 \\
\hline B & 1 & 1 & 1 & 0 \\
\hline C & 0 & 0 & 1 & 0 \\
\hline D & 1 & 0 & 1 & 0 \\
\hline E & 0 & 0 & 0 & 1 \\
\hline F & 1 & 0 & 0 & 1 \\
\hline G & 0 & 1 & 0 & 1 \\
\hline H & 1 & 1 & 0 & 1 \\
\hline I & 0 & 1 & 1 & 1 \\
\hline J & 1 & 1 & 1 & 1 \\
\hline K & 0 & 0 & 1 & 1 \\
\hline L & 1 & 0 & 1 & 1 \\
\hline M & 0 & 0 & 0 & 0 \\
\hline N & 1 & 0 & 0 & 0 \\
\hline O & 0 & 1 & 0 & 0 \\
\hline P & 1 & 1 & 0 & 0 \\
\hline
\end{tabular}

Fuente: http://bibliotecadigital.univalle.edu.co/bitstream/10893/1560/2/Rev.Ing.\% 20y\%20Competitividad\%20Vol\%205, $\% 20$ No\%202, P.63-72, 2004.pdf

Los dispositivos bidireccionales (transmitters-receivers) tienen la capacidad de responder y confirmar la correcta realización de una orden, lo que puede ser muy útil cuando el sistema X10 está conectado a una PC que muestra los estados en que se encuentra la instalación domótica de la vivienda. El número de módulo (Unit Addresses) y la función a realizar (Function Codes) se transmiten de acuerdo con la tabla 2.

TABla 2. Módulo (Unit Addresses) y Función a REAlizar (Function Codes)

\begin{tabular}{|c|c|c|c|c|c|}
\hline Unit & & Key & Codes & & \\
\hline Addresses & D1 & D2 & D4 & D8 & D16 \\
\hline 1 & 0 & 1 & 1 & 0 & 0 \\
\hline 2 & 1 & 1 & 1 & 0 & 0 \\
\hline 3 & 0 & 0 & 1 & 0 & 0 \\
\hline 4 & 1 & 0 & 1 & 0 & 0 \\
\hline 5 & 0 & 0 & 0 & 1 & 0 \\
\hline 6 & 1 & 0 & 0 & 1 & 0 \\
\hline 7 & 0 & 1 & 0 & 1 & 0 \\
\hline 8 & 1 & 1 & 0 & 1 & 0 \\
\hline 9 & 0 & 1 & 1 & 1 & 0 \\
\hline
\end{tabular}




\begin{tabular}{|c|c|c|c|c|c|}
\hline 10 & 1 & 1 & 1 & 1 & 0 \\
\hline 11 & 0 & 0 & 1 & 1 & 0 \\
\hline 12 & 1 & 0 & 1 & 1 & 0 \\
\hline 13 & 0 & 0 & 0 & 0 & 0 \\
\hline 14 & 1 & 0 & 0 & 0 & 0 \\
\hline 15 & 0 & 1 & 0 & 0 & 0 \\
\hline 16 & 1 & 1 & 0 & 0 & 0 \\
\hline All Units Off & 0 & 0 & 0 & 0 & 1 \\
\hline All Units On & 0 & 0 & 0 & 1 & 1 \\
\hline Off & 0 & 0 & 1 & 0 & 1 \\
\hline On & 0 & 0 & 1 & 1 & 1 \\
\hline Dim & 0 & 1 & 0 & 0 & 1 \\
\hline Bright & 0 & 1 & 0 & 1 & 1 \\
\hline All Ligths Off & 0 & 1 & 1 & 0 & 1 \\
\hline Extended Code & 0 & 1 & 1 & 1 & 1 \\
\hline Hail Request & 1 & 0 & 0 & 0 & 1 \\
\hline Hail Acknowledge & 1 & 0 & 0 & 1 & 1 \\
\hline Pre-set Dim & 1 & 0 & 1 & $\mathrm{X}$ & 1 \\
\hline Extended Code(Analog) & 1 & 1 & 0 & 0 & 1 \\
\hline Status $=\mathrm{On}$ & 1 & 1 & 0 & 1 & 1 \\
\hline Status=Off & 1 & 1 & 1 & 0 & 1 \\
\hline Status Request & 1 & 1 & 1 & 1 & 1 \\
\hline
\end{tabular}

Fuente: http://bibliotecadigital.univalle.edu.co/bitstream/10893/1560/2/Rev.Ing.\% 20y\%20Competitividad\%20Vol\%205, $\% 20$ No\%202, P.63-72, 2004.pdf

La tabla 3 muestra el conjunto de comandos básicos con una breve descripción.

TABLA 3. CONJUNTO DE COMANDOS

\begin{tabular}{|l|l|c|}
\hline FUNCTION & \multicolumn{1}{|c|}{ DESCRIPCION } & $\begin{array}{c}\text { BINARY } \\
\text { VALUE }\end{array}$ \\
\hline All Units Off & Desactivación de todos los módulos de luces. & 0000 \\
\hline All Lights On & Activación de todos los módulos de luces. & 0001 \\
\hline On & Activación del módulo direccionado. & 0010 \\
\hline Off & Desactivación del módulo direccionado. & 0011 \\
\hline Dim & Reducción de intensidad. & 0100 \\
\hline Bright & Aumento de intensidad. & 0101 \\
\hline Extended Code & Para transmisión de hasta 256 códigos de función adicional. & 0111 \\
\hline Extended Data Transfer & Para transmisión de bytes adicionales (por ej. conversor A/D) & 1100 \\
\hline
\end{tabular}

Fuente: http://bibliotecadigital.univalle.edu.co/bitstream/10893/1560/2/Rev.Ing.\% 20y\%20Competitividad\%20Vol\%205, \%20No\%202, P.63-72, 2004.pdf 


\section{Módulo receptor}

En la figura 4, se muestra el módulo receptor. Para un mejor desempeño en la etapa de filtrado de señal, fue necesario trabajar con la entrada de $220 \mathrm{~V}$ directamente, sin aislar, pues los transformadores convencionales tienden a atenuar grandemente la señal de $120 \mathrm{KHz}$.

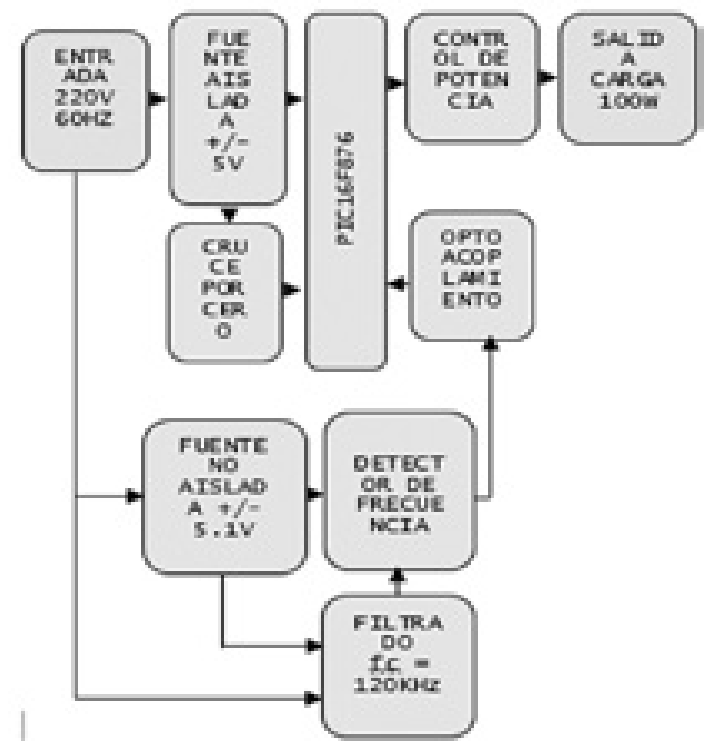

Fig. 4. Módulo receptor.

Fuente: elaboración propia.

Es por ello que se tienen dos fuentes: una aislada para el cruce por cero y el microcontrolador, y otra no aislada para el filtrado de seńal y el detector de frecuencia. La parte no aislada obtiene las señales de la red y las comunica al microcontrolador aislado por medio de un optoacoplador. Estas señales son pulsos de $1 \mathrm{~ms}$ de duración y están sincronizadas con los cruces por cero. Una vez que las órdenes son codificadas por el microcontrolador, se efectúa el control de la carga, en este caso, se pueden activar las funciones de prender o apagar.

En las figuras 5 y 6 , se observan los circuitos de cruce por cero y el circuito del microcontrolador.

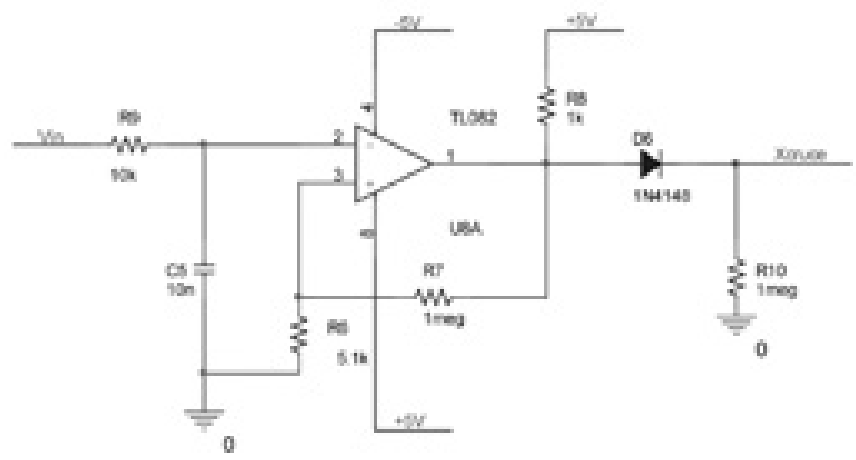

Fig. 5. Circuito de cruce por cero.

Fuente: elaboración propia. 


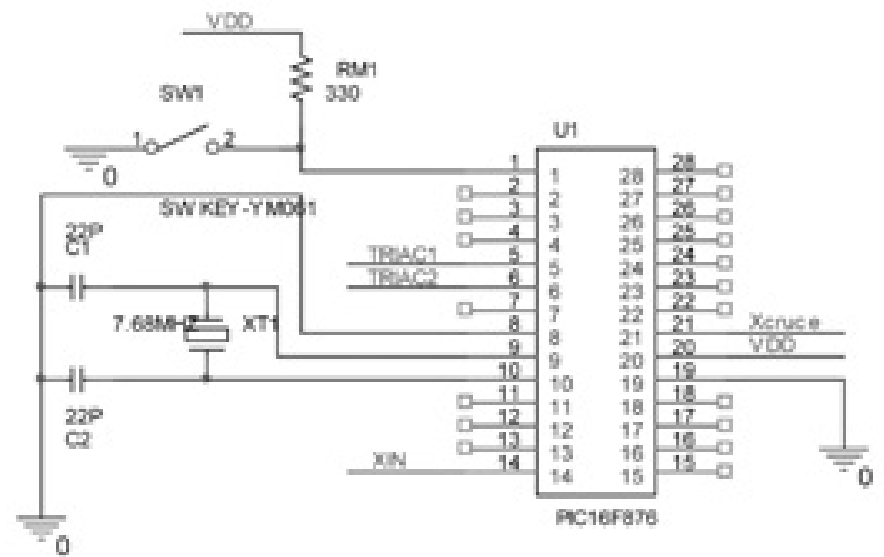

Fig. 6. Circuito microcontrolador.

Fuente: elaboración propia.

El microcontrolador puede prender o apagar una carga de $100 \mathrm{~W}$ a través de un optotriac y un triac. En la parte no aislada, se tiene la alimentación de $+/-5.1$ voltios, los cuales se obtienen de un condensador cuyo valor se calcula a partir de la corriente necesaria para la parte analógica, la que se muestra en la ecuacion 1 .

$$
\text { Irms }=220 \mathrm{~V}(2 \pi 60) \mathrm{C}
$$

El filtro, al igual que la fuente de alimentación anterior, se conecta directamente a la red. Es necesario, primero, filtrar la tensión de $60 \mathrm{~Hz}$ con un filtro Pasa altos que se calcula mediante la ecuacion 2.

$$
f o=\frac{1}{2 \pi R C}
$$

con fo $=50 \mathrm{KHz}$, se aproxima $\mathrm{R}=330 \mathrm{Ohm}$ y $\mathrm{C}=0 \mathrm{nF}$.

Luego, se limita la señal procedente del filtro a 0-5 V con diodos de señal para que pueda ingresar con seguridad al PLL detector de frecuencia que está sintonizado a $120 \mathrm{KHz}$. El PLL detecta la frecuencia y produce un pulso lógico mientras dure la señal. Esta señal se ingresa al microcontrolador por medio de un optoacoplador, como se muestra en la figura 7.

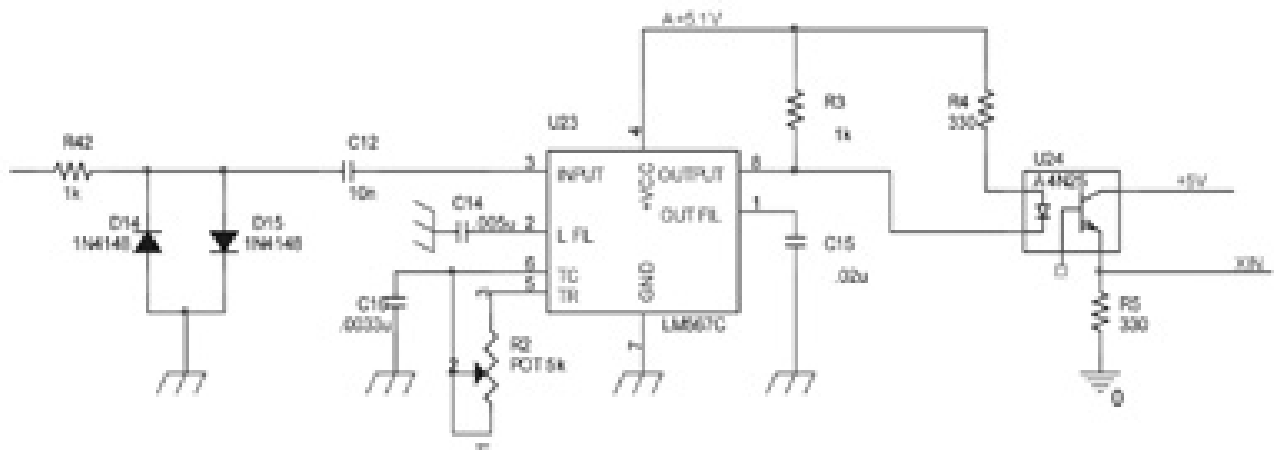

Fig. 7. Circuito de protección, sintonizado y optoacoplamiento.

Fuente: elaboración propia. 
Es necesario eliminar el ruido de la red y aportar algo de ganancia a la seńal con un filtro pasabanda. Para ello, se diseńó el filtro pasabanda Butterworth de segundo orden, con frecuencia inferior de $100 \mathrm{KHz}$ y superior de $140 \mathrm{KHz}$, y ganancia de 10. En la figura 8, se muestra la respuesta. En el circuito implementado, la ganancia está en el orden de 6 a 7.

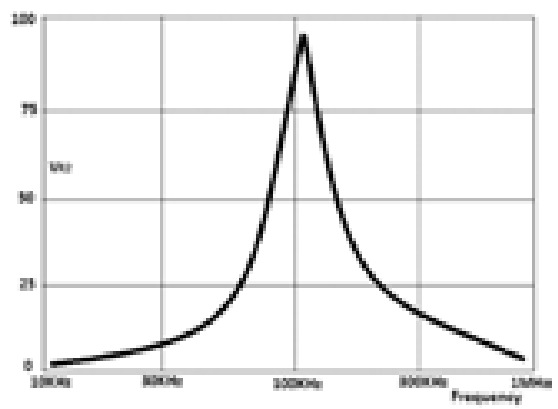

Fig. 8. Respuesta filtro Butterworth. Fuente: elaboración propia.

\section{Módulo emisor}

En la figura 9, se muestra el esquema del circuito emisor. La etapa de amplificación de corriente es necesaria, pues el optoacoplador no puede proveer la corriente necesaria para inyectar la seńal en la red. La capacidad de corriente del amplificador se obtiene a partir de un proceso de prueba y error. Se eligió un amplificador tipo $\mathrm{B}$, el cual posee rápida respuesta y una corriente máxima de $1 \mathrm{~A}$. La tensión de 7.5 V se obtiene al decidir el alcance máximo del equipo al probar, nuevamente, con varias tensiones. A más tensión de inyección, mayor alcance. Sin embargo, esta tensión no se debe elevar demasiado para prevenir la interferencia con otros equipos conectados a la red.

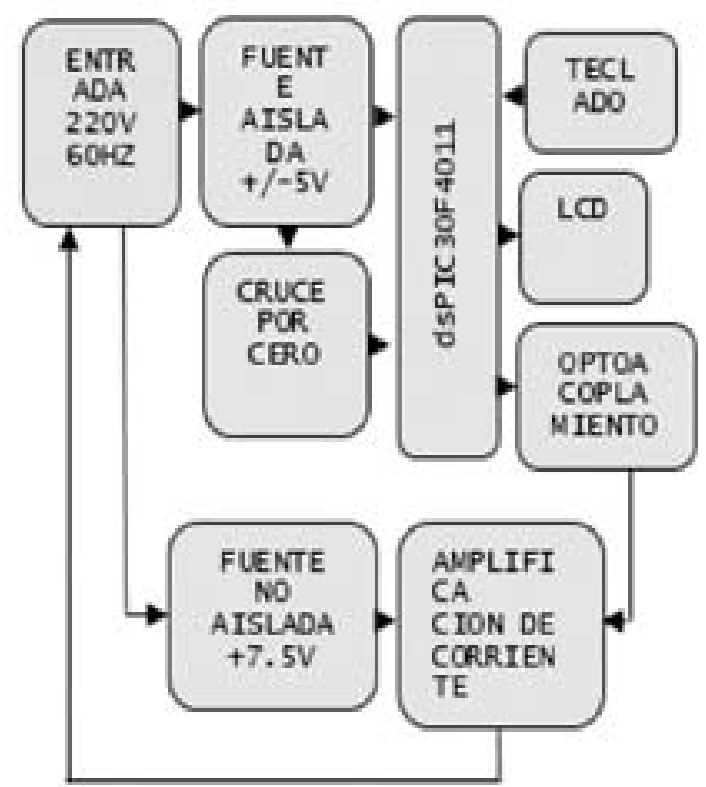

Fig. 9. Modulo emisor.

Fuente: elaboración propia. 
Se eligió el controlador dsPIC30F4011debido a su capacidad de memoria y facilidad de programación, como se muestra en la figura 10 .
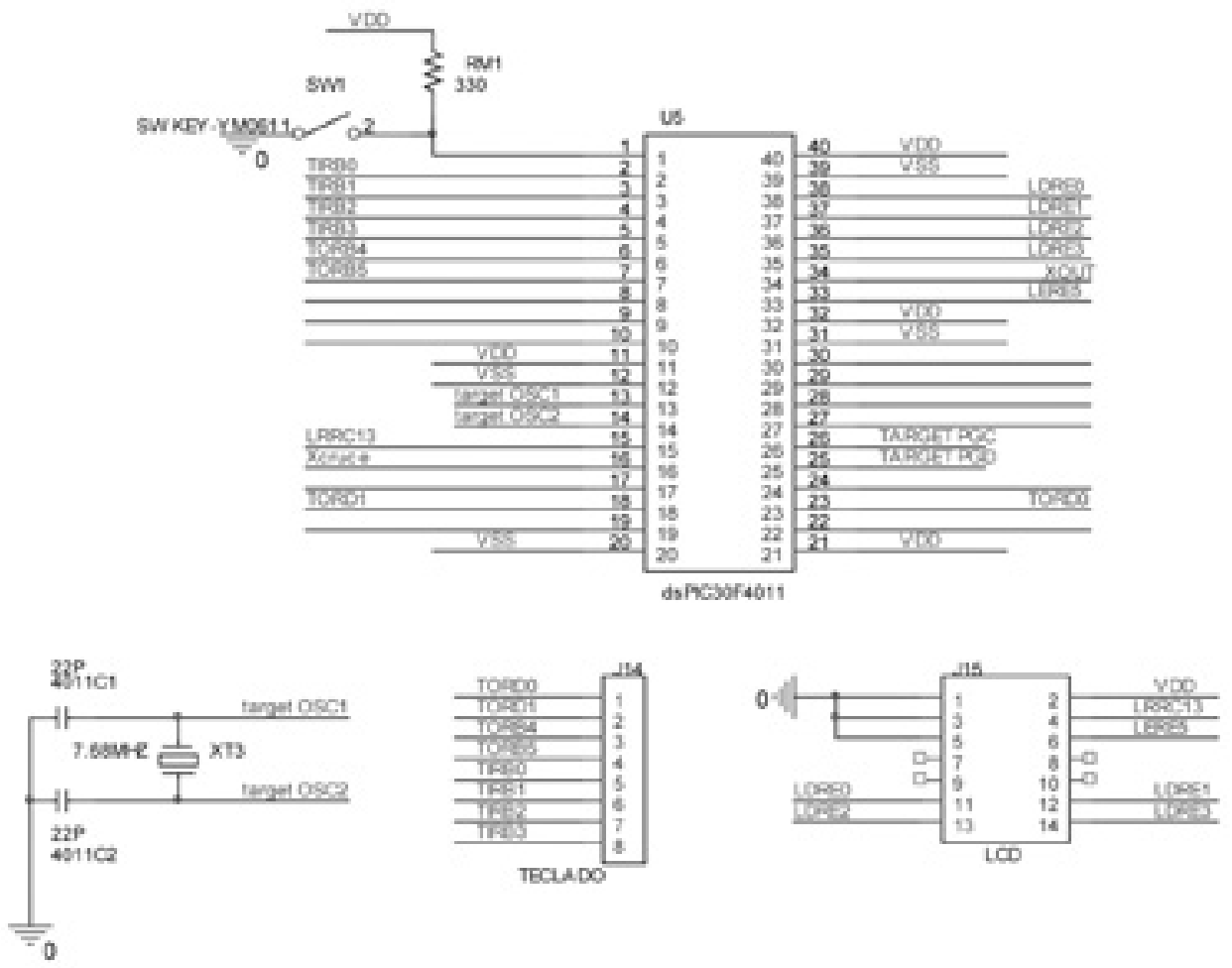

\section{DSPIC4011, TECLADO, LCD}

Fig. 10. Controlador dsPIC30F4011.

Fuente: elaboración propia.

Asimismo, en la figura 11, se muestra el circuito de transmisión.

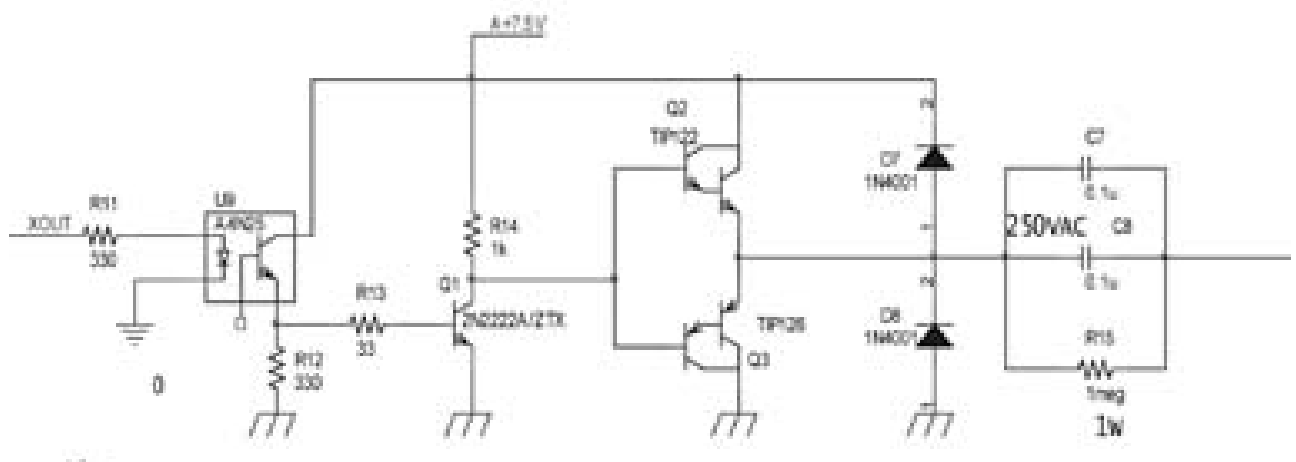

TRANSMISION

Fig. 11. Transmisión de la señal.

Fuente: elaboración propia. 


\section{Software}

En la figura 12, se muestran los diagramas de flujo tanto para el emisor como para el receptor:

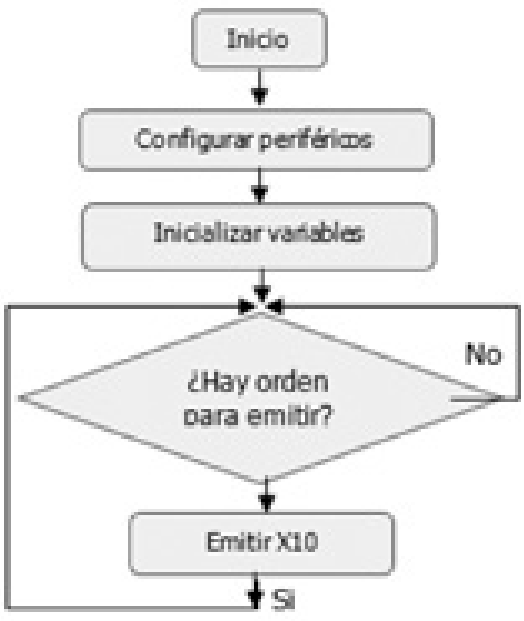

(a)

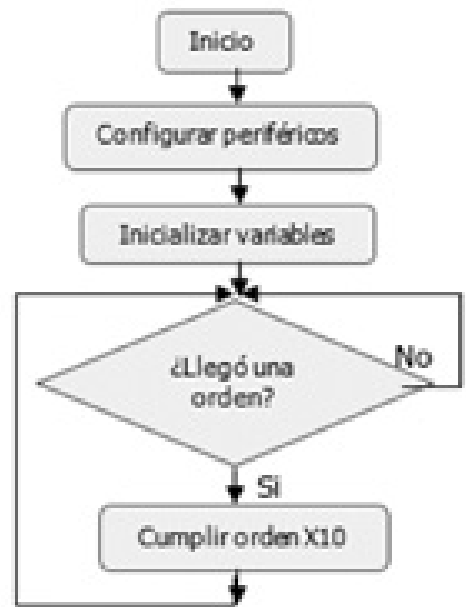

(b)

Fig. 12. Software de (a) emisor y (b) receptor.

Fuente: elaboración propia.

Cabe señalar que, para el caso de la unidad emisora, las órdenes se efectúan mediante un teclado y un LCD, y en el receptor se ejecutan las órdenes para controlar la carga.

\section{Resultados}

Se ha implementado un sistema capaz de enviar y recibir señales X10 y que, además, puede controlar cargas de acuerdo con las órdenes emitidas. Trabaja con $220 \mathrm{VAC}, 60 \mathrm{~Hz}$, aunque se puede modificar para otras tensiones. Tiene un alcance de $30 \mathrm{~m}$ y es tolerante al ruido. En la figura 13, se muestra la secuencia completa de la implementación de los módulos de transmisión y recepción de sistema X10.

Con las pruebas realizadas y los módulos implementados, se pudo verificar todos los conceptos mencionados en esta investigación de manera práctica. Como se observa en las figuras, se utilizó, para la prueba del sistema, una red real y un foco como carga de control de prueba, con lo que se obtuvo resultados satisfactorios.

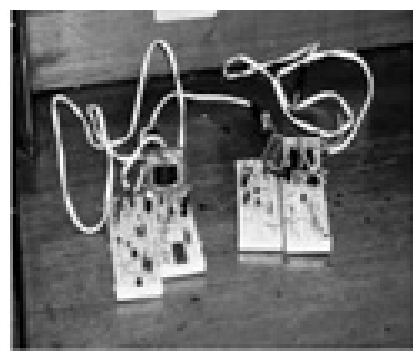

(a)

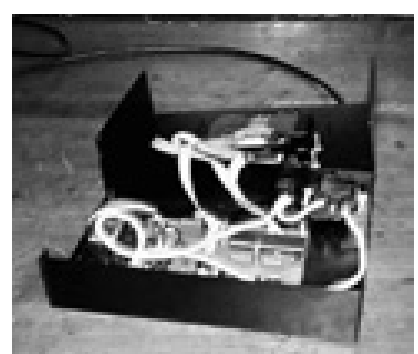

(b) 


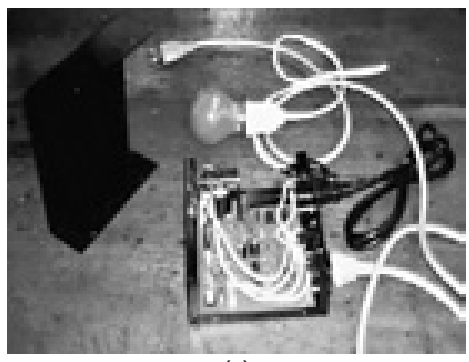

(c)

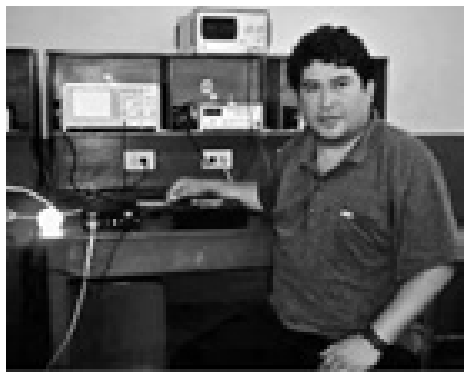

(e)

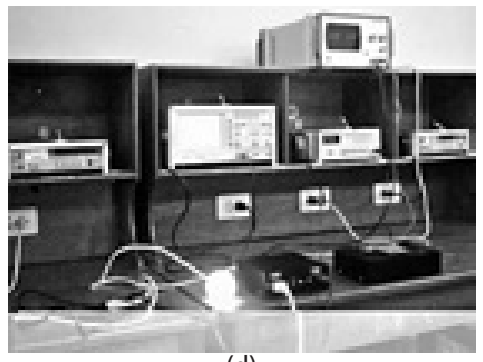

(d)

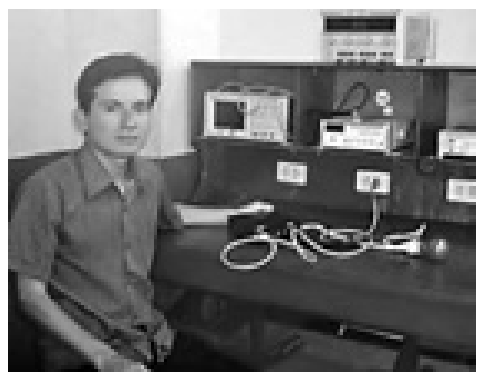

(f)

Fig. 13. Módulo X10, en OfflOn: (a) sistema en prueba; (b) sistema de transmisión en ensamblaje; (c) sistema de recepción en ensamblaje; (d) sistema completo funcionando; (e), $(f)$ autores del proyecto.

Fuente: elaboración propia.

\section{Conclusiones}

a) Se pudo verificar que sí es posible inyectar señales de alta frecuencia en la red eléctrica así como recuperarlas con circuitos electrónicos.

b) Se demostró que se pueden implementar redes de control de electrodomésticos y cualquier otro tipo de artefactos enviando y recibiendo señales a través de la red.

c) El protocolo X10, por estar estandarizado, define tanto la trama del protocolo como las especificaciones del hardware que lo soporta.

d) Es posible generar aplicaciones diversas de domótica utilizando este protocolo.

e) Se demostró que se puede aplicar este sistema en cualquier tipo de red domiciliaria o industrial sin realizar ninguna modificación a la red existente.

\section{Referencias bibliográficas}

[1] H. Paz, "Transmisión de información a través de la red eléctrica domiciliaria" [En línea]. Disponible en: http://tecnura.udistrital.edu.co/ojs/index.php/revista/article/view/97/97. [Accedido: 15-set-2003]

[2] X-10 ${ }^{\circ}$ Home Automation Using the PIC16F877A [En línea]. Disponible en: http://www.microchip.com/. [Accedido: 10-jul-2014]

[3] Vera, A. Alarcón, O. Polanco, R. Nieto y A. Bernal, "Aplicación de las Comunicaciones Inalámbricas a la Domótica”, Ingeniería y conectividad, Volumen V, número correspondiente al 2 mayo de 2004, pp. 63- 
72 [En línea]. Disponible en: http://bibliotecadigital.univalle.edu.co/bitstream/10893/1560/2/Rev.Ing.\% 20y\%20Competitividad\%20Vol\%205,\%20No\%202,P.63-72,2004.pdf. [Accedido: 10-jul-2014]

[4] Control de Domótica por Domodesk, Empresa Española. [En línea]. Disponible en: http://www.domodesk.com/. [Accedido: 15-set-2003]

[5] Casa Inteligente [En línea]. Disponible en: www.casainteligente.com/x10/x10.htm. [Accedido: 16-set-2013]

[6] Edificios Inteligentes. [En línea]. Disponible en: http://www.umanizales.edu.co/publicaciones/campos/ingenieria/ventana_informatica/html/ventana11/Domotica.pdf. [Accedido: 16-set-2013]

[7] Domotic Solution, “Domótica y hogar digital”, en Danca Intelligent Designs, 2010. [En línea]. Disponible en: http://www.domodanca.com/domotica_casasinteligentes.html. [Accedido: 10-jul-2014]

[8] Lbs, "Módulos X10". [En línea]. Disponible en: http://www.lsb.es/imagenes/x10_introduccion.pdf. [Accedido: 10-jul-2014]

[9] Módulo compatible X10 por Phillip Plunkett. Abacus Electrics. [En línea]. Disponible en: http://www. abacuselectrics.com/. [Accedido: 10-jul-2014]

[10] P. Molina. "Diseño e implementación de un sistema de seguridad e iluminación controlado remotamente desde el internet y SMS a partir de un módulo de seguridad con protocolo X10 A.", tesis para la obtención del título de Ingeniero en Electrónica y Telecomunicaciones, Facultad de Ingeniería eléctrica y Electrónica de la Universidad Politécnica Nacional, Quito-Ecuador [En línea]. Disponible en: http://bibdigital.epn.edu. ec/handle/15000/3801. [Accedido: 11-jul-2011]

[11] R. Bertuzzi, P. Guarda, J. Salazar. "Automatización del hogar usando el protocolo de Comunicación x10”. Valdivia-Chile. [En línea]. Disponible en: http://www.qsl.net/ea3cno/pdf/articulo_x10.pdf. [Accedido: 25-jun-2011]. 